\title{
Editorial: Behavioural and Ecological Consequences of Urban Life in Birds
}

\author{
Caroline Isaksson ${ }^{1 *}$, Amanda D. Rodewald ${ }^{2}$ and Diego Gil ${ }^{3}$ \\ ${ }^{1}$ Department of Biology, Lund University, Lund, Sweden, ${ }^{2}$ Cornell Lab of Ornithology and Department of Natural Resources, \\ Cornell University, Ithaca, NY, United States, ${ }^{3}$ Departamento de Ecología Evolutiva, Museo Nacional de Ciencias Naturales \\ (CS/C), Madrid, Spain
}

Keywords: biodiversity, environmental stress, pollution, species interactions, urbanization

Editorial on the research topic

Behavioural and Ecological Consequences of Urban Life in Birds

\section{INTRODUCTION}

The footprint of urban areas continues to expand across the globe, with urban land cover expected to triple between 2000 and 2030 (United Nations, 2014). This dramatic transformation from natural or less-intensively used land to impervious surfaces and buildings has altered species composition and distributions, homogenized communities, and even prompted local extinctions (Marzluff and Ewing, 2001; McKinney, 2002; Shochat et al., 2010). Because the most intense urban development is projected to occur within biodiversity hotspots (Elmqvist et al., 2013), urbanization stands to be one of the greatest threats to species persistence in the future. Yet nearly $20 \%$ of the roughly 10,000 described bird species can still be found in cities (Aronson et al., 2014). Thus, understanding the enabling factors that allow species to persist within urbanized landscapes is as important as identifying the drivers of species loss.

The present issue on "Behavioural and Ecological Consequences of Urban Life in Birds" showcases 30 articles that provide insights into species responses to urbanization through diverse lenses, including biogeography, community ecology, life history evolution, and physiology. Behavioral responses are a central theme across articles, in part because behavior often defines initial responses to urbanization and provide clues about how certain species cope with urban habitats, human presence, and novel resources (e.g., food and nest boxes) and conditions (e.g., pollution, noise, artificial light). As Topic Editors we aimed both to cover a wide range of topics and approaches and to highlight research around the world, especially those studies conducted outside of Europe and North America. We believe that it is important to cover research in the Southern Hemisphere, as this comparatively less studied area contains the highest levels of avian diversity in the world (Thomas et al., 2008). In total, 19 countries from 6 continents are represented in the articles within this issue, which collectively report on more than 700 bird species. The top-three species for urban/rural comparisons were, not surprisingly, the great tit (Parus major), the house sparrow (Passer domesticus) and the blackbird (Turdus merula). This pattern is consistent with the suggestion in the contributing paper by Ibáñez-Álamo et al., who show that urbanization not only affects the birds but also how we, researchers, study them. The more urban a species is the more frequently it is studied.

Here we review and synthesize the key findings of this issue. The articles provide a nice overview of the general advances in the field of urban ecology, especially in relation to birds. In addition, these studies tackle many still unanswered questions at both macro- and micro-ecological levels, along with phenotypic and evolutionary responses to environmental change that urgently need to be addressed to maintain avian diversity on an urbanizing planet. 


\section{Fear and Boldness}

Millions of people around the world enjoy birds through bird-feeding or bird-watching, but the reality is that the experience is not always mutually pleasant from the perspective of birds. Whereas, humans often enjoy the interaction and entice birds to come closer, birds must evaluate the risk at every encounter.

A common expectation is that urban birds should be less fearful of humans than rural counterparts. Several contributed papers address the potential fear experienced by birds by using flight initiation distance (FID) as a proxy for "fear" toward humans i.e., the shorter FID, the closer humans can approach and the more fearless is the bird. Indeed, two meta-analyses of 32 (Samia et al.) and 42 European species (Symonds et al.), respectively, report that the more urbanized a species is (i.e., longer time since colonization of cities), the shorter the FID and, presumably, the less fear is experienced by the birds. A field-intensive study of burrowing owls (Athene cunicularia) by Carrete and Tella also found that breeding pairs were more fearless toward humans in urban compared to rural habitats.

But what drives those patterns? Symonds et al. explicitly tested the extent to which encephalization (brain complexity and size) affected tolerance level of urban birds, but found no significant association between brain size and FID in this set of 42 species. Interestingly, the authors of the two metaanalyses suggest contrasting conclusions to the shorter FIDs of urban birds-released selection via reduced predation (Samia et al.) or enhanced selection of birds with low responsiveness toward humans (Symonds et al.). Sprau and Dingemanse applied a statistical approach to distinguish plastic responses from patterns of non-random distributions of behavioral variation in FID and aggression in the great tit. The striking result was that the two axes of behavior were non-randomly distributed, such that fearless (bold) birds occurred more frequently in areas with more cars but fewer humans, while fearful (shy) individuals were predominantly found in areas with fewer cars and more humans (Sprau and Dingemanse). Potential explanations for this pattern include non-random settlement, habitat- and type-specific survival, or irreversible plasticity in response to long-term exposure to urban environmental stress. The unexpected finding of shorter FID with fewer humans might be explained by vehicle noise, as Petrelli et al. found that noisy urban environments facilitated closer human approaches, and thus shorter FIDs, than more quiet habitats.

Other contributed papers explored behavior (often these assays are linked to so-called personalities or behavioral syndromes) using other approaches. Two studies examined coping responses of urban and rural great tits when handled, a widely used technique to score aggression. Senar et al. found that urban tits used a more pro-active coping strategy via more distress calls (fear screams) and higher pecking rates (aggression), whereas Charmantier et al. detected no such differences in great tits from Montepellier. Instead, the urban great tits from Montpellier were more explorative to a novel environment
(Charmantier et al.), which is consistent with the exploratory behavior that Carrete and Tella report for urban burrowing owls. Great tit aggression was also assayed in the study by Sprau and Dingemanse using staged trials with a caged mount and playback of song. As for FID (see above)-boldness increased with traffic-load.

\section{Behavioral and Fitness Consequences of Human-Provided Resources}

The novelty, availability, and predictability of resources, such as bird-feeders and nest boxes, are among the most striking ways that urban landscapes differ from non-urban areas. Yet the extent to which urban-dwelling species respond to and rely upon human-provided resources varies widely. The so-called "urban exploiters" depend on the anthropogenic resources and are often abundant within cities, whereas the "urban adapters" are more opportunistic (McKinney, 2002).

While the extent to which anthropogenic resources positively or negatively affect birds varies among species, systems, and geographies, several papers in this issue provide new insights on this respect. Anthropogenic resources, such as birdseeds and invasive fruits, may create more predictable and homogenous environments that reduce selective pressures compared to natural environments. Rodewald and Arcese corroborate this hypothesis by demonstrating reduced variability in reproductive contributions within and among-female Northern cardinals (Cardinalis cardinalis) in urban than rural environments, despite comparable variation in body condition. In this way, urban females were relatively homogeneous in terms of performance, whereas rural females spanned a wider range of high to low performers. While bird-feeding is associated with many positive outcomes for birds, Reynolds et al. remind that community responses may differ between continents and hemispheres; for example, gregarious non-native species tend to dominate at feeding tables in the Southern hemisphere. Indeed, Galbraith et al. report that 10 of the 11 species recorded using urban bird feeders in New Zealand were non-native, including the two dominating species of the house sparrow P. domesticus and the spotted dove Streptopelia chinensis. This improved benefit for invading species may explain the patterns of abundance and spreading that characterize these species.

Other articles highlight the tradeoffs between quantity and quality of urban-associated resources, particularly when urban resources are less diverse and of lower nutritional value. Isaksson et al. revealed differences in the nutritional fatty acid physiology of four common passerine species between the urban and rural habitats that were not attributed to specific foods. Rather, the fatty acid profile of urban tits (Paridae) and sparrows (Passeridae) suggest that the urban diet of these two families could affect the birds through two different pathways, inflammation and oxidative stress, respectively.

Of course, food is not the only urban resource, and many birds are attracted to nest boxes, especially when natural holes are limited. The presence of nest boxes can allow a species to colonize areas that otherwise would have been inhospitable and may boost reproductive success of certain species. At the same 
time, nest boxes may increase risk of predation if they are more readily located than natural cavities. Consistent with this idea, a meta-analysis conducted by Vincze et al. showed that artificial nests (both hole and cup nests) in cities were more likely to be depredated than natural nests, but surprisingly, vice versa in less urbanized areas. Although differences might be attributed to shifts in predator composition, abundance, or behavior between rural and urban landscapes, no ecological drivers were identified.

Apart from predation, another ecological factor that can influence breeding success in an artificial nest box is weather. Despite the careful attention given to the design and construction of nest boxes, it was surprising to learn from Duckworth et al. that nest boxes are typically less insulated than natural nest cavities and, consequently, are associated with lower survival of nestlings in inclement weather.

\section{Physiological and Behavioral Effects to Novel Abiotic Stressors}

Urbanization is commonly associated with pollution, whether due to emissions in air, artificial light at night (ALAN) or noise (e.g., Salmón et al., 2018). Indeed, Bailly et al. demonstrate that although levels of nitrogen gas $\left(\mathrm{NO}_{2}\right)$ were consistently higher in urban than in rural areas, most non-essential metals were undetectable in the blood of great tits (P. major) living in urban and rural environments.

Passive exposure (e.g., oral intake and inhalation from ambient environment) is not the only form of pollution that can affect birds. In fact, some urban birds actively seek out toxic substances, such as cigarette butts, to include in their nest. Previously, it has been shown that this reduces ectoparasite load of nestlings (Suárez-Rodríguez et al., 2012). Here the contributed paper by Suárez-Rodriguez et al. show genotoxic effects of cigarette butts on the incubating and caring parent of house finch (Carpodacus mexicanus) and house sparrow (P. domesticus) from Mexico City that led to an interesting trade-off between parasite repellence and DNA-damage. A number of papers in this issue investigate other markers of cellular stress and damage, specifically oxidative damages to protein and lipids, telomere length and gene expression of inflammatory genes between urban and rural passerines (Herrera-Duenas et al.; Isaksson et al.; Biard et al.; Capilla-Lasheras et al.). Results provided mixed evidence across species; sparrows (Passeridae) for instance, show overall more damage in relation to urbanization compared to tits (Paridae) (see also Salmón et al., 2018). For all these correlative studies the exact causal driver of the stress and damage experienced in urban birds could not be identified. In contrast, ALAN was experimentally manipulated by Welbers et al., who exposed breeding great tits to either white, green, red LED light or left dark as a control and investigated its effect on daily energy expenditure (DEE). The DEE of great tits exposed to white and green light during chick feeding was significantly lower than those exposed to the control treatment-a pattern that the authors regarded as an indirect consequence of the positive association between lights and insect abundance. This is an intriguing positive effect of an urban pollution source that may lead to balance out some of the more negative effects.
There is a growing literature of studies showing that noise pollution is an important factor affecting acoustic communication in birds, and that many bird species show modifications in song amplitude, frequency and temporal characteristics that increase the chances of song reaching receivers (Gil and Brumm, 2014). Two articles in this issue deal with acoustic communication in urban environments. The first of them, by Sewall and Davies, identifies differences between urban and rural populations in the brain expression of an early gene (FOS), a proxy for recent neural activity, indicating different neural responsiveness to stimuli that could underpin differences in behavior in the song sparrow (Melospiza melodia). Another study, by Sierro et al., shows that, in the particular noise environment of an airport, European blackbirds modify the composition of their song, but not their overall frequency characteristics, reducing the part of the song which has the lowest amplitude. In addition, birds near the airport advance the timing of their dawn chorus at the period of the season when overcraft activity overlaps most with their singing. In contrast, this advance disappears later in the season, when the natural timing of song is much earlier than the airport activity.

\section{Reproduction and Life History}

Understanding the ecological and evolutionary consequences of urbanization requires, in part, that we identify how urbanassociated factors impact fitness across phenotypes, populations, and species. Several articles reported that cities reduced breeding success via a range of pathways including clutch size, fledgling success and chick quality (Biard et al.; Capilla-Lasheras et al.; Charmantier et al.; Corsini et al.), though others found equivalent breeding performance and condition among rural and urban populations (Rodewald and Arcese).

Although reduced productivity is often attributed to predation, this pattern may also reflect a life-history strategy characterized by urban birds adopting a slower pace of life compared to rural individuals (Sepp et al., 2018). In this special issue, Charmantier et al. provide counterevidence to a slow life in the city by showing that urban great tits have behavioral phenotypes linked to a fast-pace of life, although they produce smaller clutches. Thus, urban great tits in this study seemed to be constrained during reproduction rather than these differences being the result of a proper life-history strategy. One constraint might be related to lower overall access of natural high quality foods or a mismatch between timing of breeding and available food.

Phenological shifts can also influence reproduction in urban populations. Due to the higher temperatures in the cities (i.e., heat island effect), cities often show phenological advances in spring with earlier bud burst and insect emergence. If birds are not able to respond to this environmental change the consequences for breeding can be detrimental (Visser and Both, 2005). Here, however, a study on great tits showed that urban birds advance their breeding which is in line with the presumed earlier phenology of a city (Charmantier et al.). In contrast, the breeding performance of the black sparrowhawk (Accipiter melanoleucus), but not the timing of breeding per se, showed different seasonal patterns in urban and rural areas, declining 
across the season in cities but improving in rural habitats (Rose et al.). The black sparrowhawks have relatively recently colonized the urban habitats of Cape Town, and thus the seemingly selective advantages of earlier breeding in the city may have not arisen yet. In the contributed paper by Fudickar et al. the underlying mechanism of advancing the timing of breeding is investigated in male dark-eyed juncos (Junco hyemalis). By comparing sedentary urban birds with migratory individuals, they show that the earlier timing of breeding in the urban population is facilitated by earlier increase in upstream baseline activity of the hypothalamic-pituitary-gonadal (HPG) axis and an earlier release of gonodal suppression affecting testosterone production (Fudickar et al.).

\section{Biodiversity and Conservation in Urban Habitats}

Urbanization stands out as one of the most important threats to biodiversity on our planet. Several articles in the special issue examined this threat explicitly, and most contributors at least alluded to the conservation implications of their findings. Focusing on the Seattle Metropolitan area in the US, Shryock et al. showed that relationships between avian species richness and vegetative productivity, as measured by the Normalized Difference Vegetation Index (NDVI), are mediated by urban development. Specifically, they found that species richness declined with NDVI within areas undergoing active development, but less so in areas of established housing development and forested reserves. Urbanization also may affect the functional diversity of avian communities in ways that might have ecosystem-level consequences. Oliveira Hagen et al. compared avian functional diversity of 25 urban areas using 27 traits from more than 500 species. Interestingly, they found that avian functional diversity is higher in cities than in seminatural habitats, a pattern attributed to higher functional habitat diversity in cities compared to single habitats within more natural landscapes. Both studies suggest that city planners can moderate the effect of urbanization on the avian community by maintaining or restoring diverse and heterogeneous native vegetation. Habitat management also may facilitate species movements and dispersal, which can be constrained in urban systems even for highly mobile organisms, as shown by Evans et al.

The altered habitat structure and the simplified avifauna composition of urban habitats change the life conditions of many species and the interspecific interactions among them, something that can strongly benefit some species whereas other species will suffer. A species that has benefited from these modifications is the North American brown-headed cowbird (Molothrus ater). This is a brood parasitic species that has increased in population densities with deforestation. Two contributed papers raise the concern of the increased abundance of this species for other

\section{REFERENCES}

Aronson, M. F., La Sorte, F. A., Nilon, C. H., Katti, M., Goddard, M. A., Lepczyk, C. A., et al. (2014). A global analysis of the impacts of urbanization on bird and plant diversity reveals key anthropogenic urban-dwelling birds. In a first study by Stiles et al. it is shown that cowbirds together with humans and feral dogs have a direct negative effect on several native bird species. The second paper by Ladin et al. uses a modeling approach to test how the negative trend of cowbirds on the population growth of the forest umbrella species, the wood trush (Hylocichla mustelina) could be averted. Their models suggest that removing cowbirds along with reforestation could stop the decline of the wood thrush.

\section{CONCLUSION}

This special issue highlights a few overarching themes among studies of urban birds, and the large number of articles reflect the great interest and timeliness of the topic. Many of the contributed studies involve a comparison of two single populations - one rural and one urban. Though comparative studies are certainly useful in identifying possible mechanisms and patterns, unreplicated designs provide limited inference about mechanisms and the variability in outcomes, as well as the underlying drivers of the effects and variabilities. Identifying drivers is challenging given that cities differ from rural habitats in a many respects, just as cities differ from each other. Although experiments are typically difficult to conduct, we would like to stress the need to envisage manipulations whenever possible to do so.

Taken together, the articles in this special issue highlight the wide variety of responses-both positive and negativeto urbanization and the challenge that biologists face in trying to generalize. The differential resilience of species in the face of urbanization changes community composition and affects interspecific relationships, thus fuelling new feedback mechanisms that add complexity to the final picture. Thus, the field of urban avian ecology have many challenges ahead and intriguing new venues for future research.

\section{AUTHOR CONTRIBUTIONS}

All three authors have made substantial work with the topic issue and with the present editorial piece.

\section{FUNDING}

DG was funded while editing this special issue by a research grant from the Ministerio de Economía y Competitividad (CGL201455577R).

\section{ACKNOWLEDGMENTS}

We wish to thank all the contributing authors for their efforts to make this issue a success. We also wish to thank the team working for Frontiers in Ecology \& Evolution for their patience and their support.

drivers. Proc. R. Soc. B 281:20133330. doi: 10.1098/rspb.2013 .3330

Elmqvist, T., Fragkias, M., Goodness, J., Güneralp, B., Marcotullio, P. J., McDonald, R. I., et al. (2013). Urbanization, Biodiversity and Ecosystem Services: Challenges and Opportunities. New York, NY: Springer. 
Gil, D., and Brumm, H. (2014). "Acoustic communication in the urban environment: patterns, mechanisms, and potential consequences of avian song adjustments," in Avian Urban Ecology, eds D. Gil and H. Brumm (Oxford: Oxford University Press), 69-83.

Marzluff, J. M., and Ewing, K. (2001). Restoration of fragmented landscapes for the conservation of birds: a general framework and specific recommendations for urbanizing landscapes. Restorat. Ecol. 9, 280-292. doi: 10.1046/j.1526-100x.2001.009003280.x

McKinney, M. L. (2002). Urbanization, biodiversity, and conservation. Bioscience 52, 883-890. doi: 10.1641/0006-3568(2002)052[0883:UBAC]2.0.CO;2

Salmón, P., Stroh, E., Herrera-Dueñas, A., von Post, M., and Isaksson, C. (2018) Oxidative stress in birds along a NOx and urbanisation gradient: an interspecific approach. Sci. Total Environ. 622-623, 635-643. doi: 10.1016/j.scitotenv.2017.11.354

Sepp, T., McGraw, K. J., Kaasik, A., and Giraudeau, M. (2018). A review of urban impacts on avian life-history evolution: does city living lead to slower pace of life? Glob. Chang. Biol. 24, 1452-1469. doi: 10.1111/gcb.13969

Shochat, E., Lerman, S. B., Anderies, J. M., Warren, P. S., Faeth, S. H., and Nilon, C. H. (2010). Invasion, competition, and biodiversity loss in urban ecosystems. BioScience 60, 199-208. doi: 10.1525/bio.2010.60.3.6

Suárez-Rodríguez, M., López-Rull, I., and Garcia, C. M. (2012). Incorporation of cigarette butts into nests reduces nest ectoparasite load in urban birds: new ingredients for an old recipe? Biol. Lett. 9:20120931. doi: 10.1098/rsbl.2012.0931
Thomas, G. H., Orme, C. D. L., Davies, R. G., Olson, V. A., Bennett, P. M., Gaston, K. J., et al. (2008). Regional variation in the historical components of global avian species richness. Glob. Ecol. Biogeogr. 17, 340-351. doi: 10.1111/j.1466-8238.2008.00384.x

United Nations (2014). World Urbanization Prospects: The 2014 Revision (United Nations, Department of Economic and Social Affairs, Population Division, New York). Available online at: www.un.org/en/development/desa/publications/ 2014-revision-world-urbanization-prospects.html

Visser, M. E., and Both, C. (2005). Shifts in phenology due to global climate change: the need for a yardstick. Proc. R. Soc. B 272, 2561-2569. doi: $10.1098 / \mathrm{rspb} .2005 .3356$

Conflict of Interest Statement: The authors declare that the research was conducted in the absence of any commercial or financial relationships that could be construed as a potential conflict of interest.

Copyright (c) 2018 Isaksson, Rodewald and Gil. This is an open-access article distributed under the terms of the Creative Commons Attribution License (CC $B Y)$. The use, distribution or reproduction in other forums is permitted, provided the original author(s) and the copyright owner are credited and that the original publication in this journal is cited, in accordance with accepted academic practice. No use, distribution or reproduction is permitted which does not comply with these terms. 